OPEN ACCESS

Edited by:

Yvette Mándi,

University of Szeged, Hungary

Reviewed by:

Delia Hoffmann,

Catholic University of Louvain,

Belgium

Erno Duda,

University of Szeged, Hungary

${ }^{*}$ Correspondence:

Ciriana Orabona

ciriana.orabona@unipg.it

${ }^{\dagger}$ These authors share first authorship

Specialty section:

This article was submitted to Inflammation,

a section of the journal

Frontiers in Immunology

Received: 24 May 2021

Accepted: 19 July 2021

Published: 30 July 2021

Citation:

Mondanelli G, Albini E, Orecchini E, Pallotta MT, Belladonna ML, Ricci G, Grohmann U and Orabona C (2021)

Pathogenetic Interplay Between IL-6 and Tryptophan Metabolism in an

Experimental Model of Obesity.

Front. Immunol. 12:713989.

doi: 10.3389/fimmu.2021.713989

\section{Pathogenetic Interplay Between IL-6 and Tryptophan Metabolism in an Experimental Model of Obesity}

\author{
Giada Mondanelli ${ }^{1 \dagger}$, Elisa Albini ${ }^{1 \dagger}$, Elena Orecchini ${ }^{1}$, Maria Teresa Pallotta ${ }^{1}$, \\ Maria Laura Belladonna ${ }^{1}$, Giovanni Ricci ${ }^{2}$, Ursula Grohmann ${ }^{1}$ and Ciriana Orabona ${ }^{1 *}$ \\ ${ }^{1}$ Department of Medicine and Surgery, University of Perugia, Perugia, Italy, ${ }^{2}$ Service Center for Pre-clinical Research, \\ University of Perugia, Perugia, Italy
}

Obesity is a metabolic disease characterized by a state of chronic, low-grade inflammation and dominated by pro-inflammatory cytokines such as IL-6. Indoleamine 2,3-dioxygenase 1 (IDO1) is an enzyme that catalyzes the first step in the kynurenine pathway by transforming L-tryptophan (Trp) into L-kynurenine (Kyn), a metabolite endowed with antiinflammatory and immunoregulatory effects. In dendritic cells, IL-6 induces IDO1 proteasomal degradation and shuts down IDO1-mediated immunosuppressive effects. In tumor cells, IL-6 upregulates IDO1 expression and favors tumor immune escape mechanisms. To investigate the role of IDO1 and its possible relationship with IL-6 in obesity, we induced the disease by feeding mice with a high fat diet (HFD). Mice on a standard diet were used as control. Experimental obesity was associated with high IDO1 expression and Kyn levels in the stromal vascular fraction of visceral white adipose tissue (SVF WAT). IDO1-deficient mice on HFD gained less weight and were less insulin resistant as compared to wild type counterparts. Administration of tocilizumab (TCZ), an IL-6 receptor (IL-6R) antagonist, to mice on HFD significantly reduced weight gain, controlled adipose tissue hypertrophy, increased insulin sensitivity, and induced a better glucose tolerance. TCZ also induced a dramatic inhibition of IDO1 expression and Kyn production in the SVF WAT. Thus our data indicated that the IL-6/IDO1 axis may play a pathogenetic role in a chronic, low-grade inflammation condition, and, perhaps most importantly, IL-6R blockade may be considered a valid option for obesity treatment.

Keywords: experimental obesity, tryptophan metabolism, indoleamine 2, 3 dioxygenase 1 (IDO1), tocilizumab (TCZ), white adipose tissue (WAT), IL-6 receptor (IL-6R), high fat diet (HFD)

\section{INTRODUCTION}

IL-6 is a pleiotropic cytokine that modulates a diverse array of functions relevant to hematopoiesis, tissue homeostasis, metabolism, and immunity (1). Its deregulation is associated with several diseases, including chronic inflammation, autoimmune disorders, and cancer. Inflammatory arthritis can indeed be successfully treated with tocilizumab (TCZ), a monoclonal antibody capable of binding and blocking the IL-6R subunit of the IL-6 receptor (2). In cancer, IL-6 drives proliferation, survival, invasiveness, and metastasis of tumor cells, while strongly suppressing the anti-tumor immune response (3). 
Indoleamine 2,3-dioxygenase 1 (IDO1) is an enzyme that catalyzes the first, rate-limiting step in the kynurenine pathway, leading to depletion of the essential amino acid L-tryptophan (Trp) and production of a series of immunoregulatory molecules collectively known as kynurenines $(4,5)$. Both effects - namely, Trp starvation and kynurenine (Kyn) production - are involved in the generation of regulatory T cells (6). Highest IDO1 expression is detectable in dendritic cells (DCs), especially in the presence of IFN- $\boldsymbol{\gamma}$ (4). In contrast, the presence of a microenvironment dominated by IL- 6 favors IDO1 targeting for proteasomal degradation via recruitment of the E3 ubiquitin ligase complex (7). Therefore, in contrast to IFN- $\gamma$, IL- 6 reduces IDO1 half-life, thus interrupting immunosuppressive mechanisms and favoring a pro-inflammatory phenotype in the DCs. However, in human cancer [in which IDO1 is often overexpressed (8)], IL-6 sustains constitutive IDO1 expression (9). Moreover, inhibition of IL-6 production by tumor cells reduces IDO1 expression and tumormediated immunosuppressive effects (9).

Obesity is a metabolic disorder characterized by a chronic, low-grade inflammatory state and associated with the development of numerous comorbid conditions, including insulin resistance and type 2 diabetes (10). The inflammatory program is activated early in adipose expansion and during chronic obesity, permanently skewing the immune system to a pro-inflammatory phenotype characterized by $\mathrm{M} 1$ macrophages and the production of IL- $1 \beta$, IL- 6 , IFN- $\gamma$, and TNF- $\alpha$ (11). Interestingly, the chronic, low-grade inflammation associated to obesity also promotes the development of numerous tumors, such as liver and colorectal cancer (12). Unexpectedly, in a previous study, mice fed with a high fat diet (HFD) and lacking IDO1 expression gained less weight, had a lower fat mass and better glucose tolerance (13). Depletion of IDO1 was found to increase the production of protective Trp metabolites by gut bacteria. Consistent with the observation in mice, obese patients have lower Trp and higher Kyn in plasma (14).

In the present study, we investigated the possible relationship between IL- 6 and IDO1 in obesity. To do so, we resorted to HFD-fed mice and found that (i) IDO1 and Kyn production increase in the stromal vascular fraction of visceral white adipose tissue (SVF WAT) along weight gain, increased fat mass, and reduced glucose tolerance and insulin sensitivity; (ii) administration of TCZ abrogates IDO1 expression and Kyn production in SVF WAT, greatly reduces weight gain and adipose tissue hypertrophy, increases insulin sensitivity, and induces a better glucose tolerance. Therefore, our data indicated the existence of an aberrant interplay between IL-6 and IDO1 in obesity and the possibility to use IL-6R blockers for therapeutic purposes in obese patients.

\section{MATERIALS AND METHODS}

\section{Mice and In Vivo Treatments}

Six- to eight-week-old male C57BL/6 mice were obtained from Charles River Breeding Laboratories and used for pharmacological studies. Ido $1^{-/-}$C57BL/6 mice were obtained from an internal breeding at the Plaisant S.r.l. animal facility. All animal studies were approved by the Italian Ministry of Health. Mice were fed with either a standard diet (SD) (Mucedola Srl) or high fat diet (HFD) containing $42 \%$ fat (Mucedola Srl). HFD was started at 8 weeks of age and continued for $10 \mathrm{wk}$ or less with ad libitum access to water and food. Daily food intake was determined at 8 a.m. by weighing the metal cage top, including the food. The average WAT weight per mouse was determined by the ratio of the total weight of the visceral WAT isolated from mice to the number of mice analyzed in each experimental group. Six to eight mice were used in each treatment or control group. Impairment of glucose homeostasis was investigated by intraperitoneal (i.p.) glucose tolerance testing (IPGTT) at specific time points of HFD feeding. Briefly, 16-h fasted mice were administered i.p. with $1 \mathrm{~g} / \mathrm{kg}$ D-glucose. Blood glucose concentrations were measured before anesthesia by tail incision using a digital glucometer (Roche). TCZ (Chugai Pharmaceutical Co.) or saline was administered i.p. at the dose of $5 \mathrm{mg} / \mathrm{kg}$ (15) every other day for $4 \mathrm{wk}$, in parallel with the dietinduced feeding, or twice a week for $6 \mathrm{wk}$, when the drug treatment was delayed $2 \mathrm{wk}$ later the starting of HFD diet. Animals were sacrificed after anesthesia by i.p. administration of Avertin (125 mg/kg) for ex vivo analyses.

\section{Isolation of SVF and Morphometry of Adipose Tissues}

Visceral white adipose tissue (WAT) was excised from mice and processed for SVF cell isolation as described (16). Briefly, tissues were cut into small pieces and digested in $1 \mathrm{mg} / \mathrm{ml}$ collagenase $\mathrm{P}$ (Roche) in HBSS for $40 \mathrm{~min}$ at $37^{\circ} \mathrm{C}$. The digested tissues were passed through a $100-\mu \mathrm{m}$ cell strainer to remove debris. After centrifugation, the floating cell layer and supernatant were removed and the cell pellet was washed with HBSS. Primary SVF cells were maintained in DMEM plus 10\% FCS. For histology, 3-4 $\mu \mathrm{m}$ of paraffin-embedded sections of WAT were stained with hematoxylin and eosin and analyzed by light microscopy. For quantification of adipocyte size, sections were analyzed by a DM2500 Leica microscope equipped with Leica DFC420C digital camera (Leica microsystem). Adipocyte diameters were measured in 30 adipocytes per section (five sections for each WAT sample), and data analysis was performed using Leica Application Suite (LAS v3.8, Leica microsystems) for digital image processing.

\section{Determination of Insulin Sensitivity in Primary Hepatocytes}

Insulin sensitivity was evaluated in primary hepatocytes isolated from mice euthanized at the end of the experiment. Specifically, the liver was cut into small pieces and perfused with a digestion medium containing $0.8 \mathrm{mg} / \mathrm{mL}$ of collagenase type IV (SigmaAldrich) in $\mathrm{HBSS}$ for $40 \mathrm{~min}$ at $37^{\circ} \mathrm{C}$. Hepatocytes were dispersed in the medium using a pipette and filtered through a $100-\mu \mathrm{m}$ cell strainer. After centrifugation, cells were washed with HBSS and kept in a serum-free medium for $1 \mathrm{~h}$ at $37^{\circ} \mathrm{C}$ before insulin stimulation. Hepatocytes were treated with $100 \mathrm{nM}$ of insulin (Sigma-Aldrich) and incubated at $37^{\circ} \mathrm{C}$ for $5,15,30$, and 60 
minutes. Cells were then washed with ice-cold PBS and lysed with ice-cold RIPA buffer (50 mM Tris- $\mathrm{HCl} \mathrm{pH} \mathrm{7.4,} 150 \mathrm{mM}$ $\mathrm{NaCl}, 1 \%$ Nonidet P-40, 0.25\% Na-deoxycholate) supplemented with Halt Protease inhibitor and Halt Phosphatase Inhibitor Cocktail (Thermo Scientific ${ }^{\mathrm{TM}}$ ). Cell lysates were immediately analyzed by immunoblot.

\section{Western Blot Analyses}

These procedures were done as described (17-19). Briefly, protein lysates were subjected to SDS-PAGE and electro-blotted onto 0.2 $\mu \mathrm{m}$ nitrocellulose membranes (Bio-Rad). Membranes were blocked with $5 \%$ non-fat dried milk in TBS and probed with a primary antibody specific for the protein of interest in combination with an appropriate horseradish peroxidaseconjugated antibody (Millipore), followed by enhanced chemiluminescence (ECL) (Bio-Rad). IDO1 was investigated with a rabbit monoclonal anti-mouse IDO1 antibody (cv152) (20) in SVF WAT cells. Akt and its phosphorylated form were revealed by specific anti-Akt and -pAkt (Ser 473) antibodies (Cell Signaling) in primary hepathocytes. Anti- $\beta$-tubulin (SigmaAldrich) was used as a normalizer.

\section{Kynurenine and Cytokine Determinations}

IDO1 activity was measured in terms of the ability to metabolize Trp to Kyn. Briefly, SVF WAT cells, at the concentration of $1.5 \mathrm{x}$ $10^{6}$ cells $/ \mathrm{ml}$, were mantained in DMEM plus $10 \% \mathrm{FCS}$ at $37^{\circ} \mathrm{C}$ in a humidified $7 \% \mathrm{CO}_{2}$ incubator. Kyn concentration in the culture supernatants was measured by high performance liquid chromatography after $24 \mathrm{~h}$ of incubation $(21,22)$. Mouse cytokines (IL-1 $\beta$, IL-4, IL-6, IL-10, IL-17A, IFN- $\gamma$, TGF- $\beta$, and TNF- $\alpha$ ) were measured in $24-\mathrm{h}$ SVF WAT culture supernatants by ELISA using specific kits (eBioscience and Thermo Fisher Scientific) and according to the manufacturer's recommendations.

\section{Real-Time PCR}

Real-Time PCR (for mouse Ido1, Ucp1, and Gapdh) analyses were carried out as described (17-19). Briefly, total RNA was extracted from SVF cells by TRIzol (Invitrogen) and reverse transcribed to cDNA with QuantiTect Reverse Transcription Kit (Qiagen). Real-time PCR was performed using SYBR Green detection and the following specific primers were used: Ido1, 5'- GATGTTCGAAAGGTGCTGC-3' and 5'-GCAGGAG AAGCTGCGATTTC-3'; Ucp 1, 5' - TCA GGATTGG CCTCTACGAC-3' and 5'-TGCCACACCTCCAGTCATTA-3'; Gapdh, 5'-CTGCCCAGAACATCATCCCT-3' and 5'-ACT TGG CAG GTT TCT CCA GG-3'. Values (means \pm SD of triplicate determination) were expressed as the ratio of Gapdhnormalized transcript expression in SVF cells from HFD-fed mice to Gapdh-normalized transcript expression in SVF cells from SD-fed mice (calibrator, in which the fold change $=1$; dotted line).

\section{Statistical Analyses}

Data are expressed as means, and error bars indicate standard deviation. At least three biological replicates were used for each measurement. The exact number of biological replicates for a specific experiment is indicated in the figure legends. A "biological replicate" is a mouse for in vivo studies. A single value for a biological replicate could be the average of values from technical replicates of the same biological replicate, but statistical comparisons were made for averages of values from biological replicates. All statistical analyses were performed using Prism version 6.0 (GraphPad Software). Data were analyzed by two-tailed unpaired Student's $t$ test or 2-way ANOVA followed by post hoc Bonferroni's test, when three or more samples were under comparison, respectively. Differences were considered significant with $p<0.05$. Data are representative of two-three independent experiments.

\section{RESULTS}

We first examined HFD-fed mice in our setting in terms of several parameters typical of obesity, such as weight gain, daily food intake, and glucose tolerance. Mice fed with SD were used as control. We focused the analysis on WAT in terms of adipocyte hypertrophy and weight. Moreover, we measured the production of cytokines by SVF WAT cells [mainly containing macrophages, hematopoietic progenitor cells (21), and adipocyte precursor cells (22)]. Starting from 2 wk of feeding, mice on HFD showed significantly higher weights, which further increased over time reaching a gain of approximately $18 \mathrm{~g}$ in $10 \mathrm{wk}$ (Figure 1A). At $10 \mathrm{wk}$ of feeding, obese mice were characterized by a significant higher daily food intake (Figure 1B), WAT adipocyte diameter (Figure 1C), and weight (Figure 1D). Moreover, at the same time, obese mice exhibited higher blood glucose concentrations when challenged with the glucose tolerance test (Figure 1E). The cytokine profile of SVF WAT cells revealed a significantly higher release of proinflammatory IL- $1 \beta$, IL- 6 , IFN- $\gamma$, and TNF- $\alpha$ but not of IL- 4 , IL10, IL-17A, and TGF- $\beta$ in HFD-fed mice (Figure 1F).

In order to evaluate IDO1 expression and activity in our setting, levels of IDO1 transcript and protein as well as release of Kyn, the main IDO1 product, were evaluated in SVF WAT cells. Results showed that, at $10 \mathrm{wk}$ of feeding, obese mice expressed a 6-fold increase in Ido1-encoding transcripts (Figure 2A) and 2fold in IDO1 protein expression (Figures 2B, C). Kyn release also increased 3-fold in the same SVF WAT cells (Figure 2D). We next compared the obesity parameters in wild-type (WT) and $I d o 1^{-/-}$mice, both fed with HFD. In agreement with previous data (13), results showed that IDO1-deficient mice gain significantly less weight (Figure 2E), and have a better glucose tolerance (Figure $\mathbf{2 F}$ ), but a reduced adipocyte hypertrophy could not be observed (Figure 2G).

Because IL-6 is a cytokine widely recognized to play a major role in obesity and is also known to exert dichotomic effects on IDO1 expression $(7,13)$, we investigated the possible effect of the cytokine on IDO1 expression and activity in the WAT of dietinduced obese mice. To do so, we resorted to TCZ, a monoclonal antibody blocking the activation of the IL-6 receptor already used by us in nonobese diabetic mice (15). More specifically, WT mice 


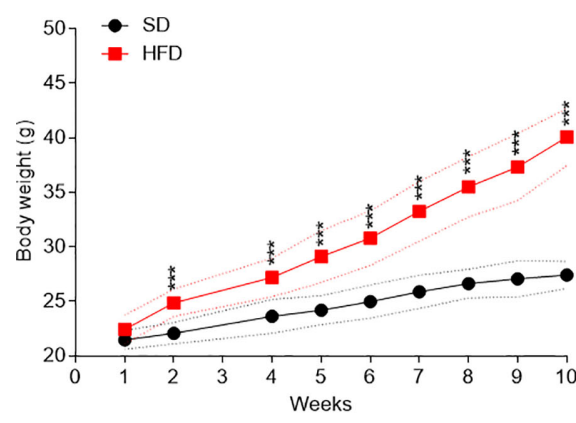

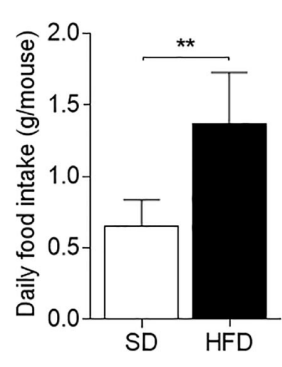

C
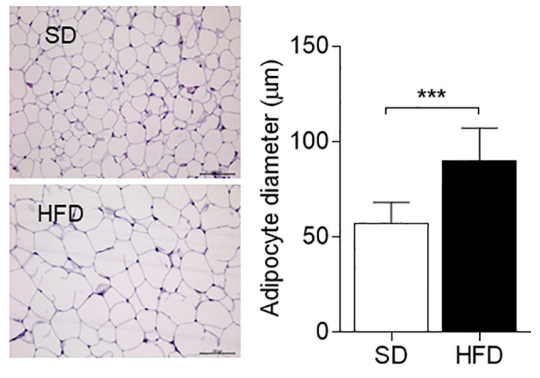

D

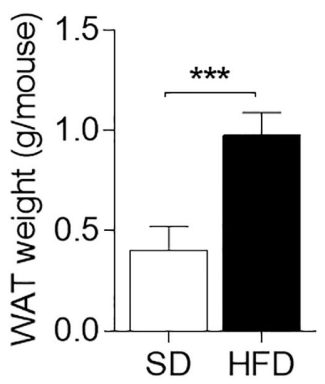

E

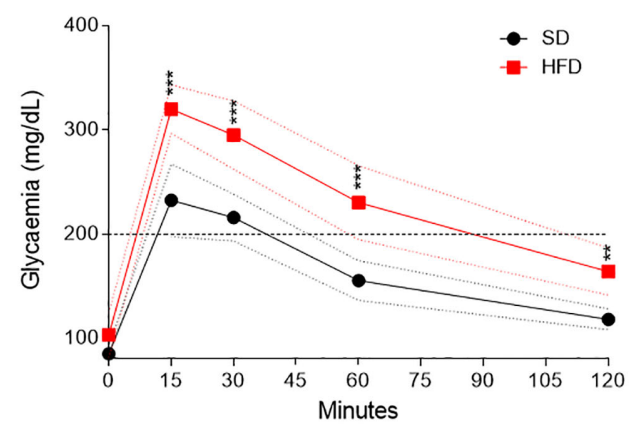

F
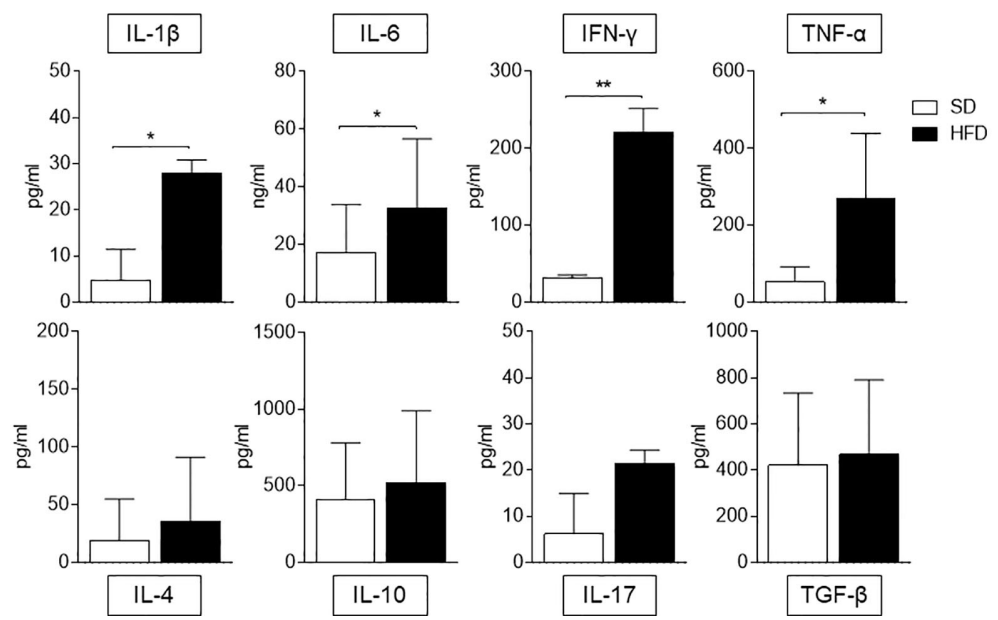

TGF- $\beta$

FIGURE 1 | Obesity and inflammatory parameters of HFD-fed mice. (A) Body weight (g) of 6-wk male mice fed with high-fat diet (HFD, $n=10$ ) for 10 wk compared with gender- and age-matched controls fed with a standard diet (SD, $n=10)$. (B) Average food intake (g) per mouse per day ( $n=10$, from two independent experiments). (C) Hematoxylin and eosin staining of visceral WATs (left panel, scale bars of $100 \mu$ m.). Analysis of adipocyte diameter (right panel). (D) Average WAT weight (g) per mouse ( $n=5$, from two independent experiments). (E) Intraperitoneal glucose tolerance test (IPGTT) after 10 weeks of HFD ( $n=5$, from two independent experiments). Glycaemia (mg/dl) was measured at different time points $(0,15,30,60$, and 120 min) from the administration of glucose. (F) Levels of cytokines secreted by SVF WAT cells in 24-h culture supernatants. Results are represented as means \pm S.D $(n=3$ biological replicates, from two independent experiments). ${ }^{\star} p<0.05,{ }^{\star \star} p<0.01,{ }^{\star \star \star} p<0.001$ HFD versus SD, two-tailed unpaired Student's $t$ test and multiple Student's $t$ test per row, corrected by post hoc Sidak-Bonferroni's method.

on HFD were administered i.p. with TCZ at the dose of $5 \mathrm{mg} / \mathrm{kg}$ every other day for $4 \mathrm{wk}$ (15). Saline injection and TCZ treatment of SD fed mice were used as controls. We observed that TCZ treatment completely abrogated IDO1 expression in terms of transcripts (Figure 3A), protein (Figures 3B, C), and Kyn release (Figure 3D) in SVF WAT cells of HFD-fed mice at the end of the feeding. No IDO1 modulation was observed in the SVF WAT cells of the TCZ-treated mice on SD, thus suggesting a dominant role of IL-6 in upregulating IDO1 in the adipose tissue of obese animals. Perhaps most impressively, TCZ administration rendered the effects of HFD similar to those of a standard diet. Indeed, no weight gain (Figure 4A) and adipocyte hypertrophy 


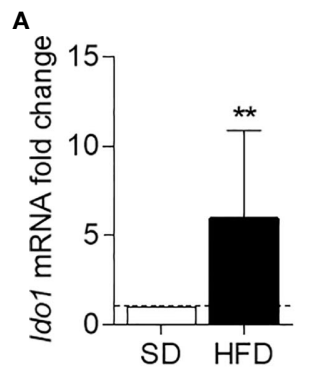

B

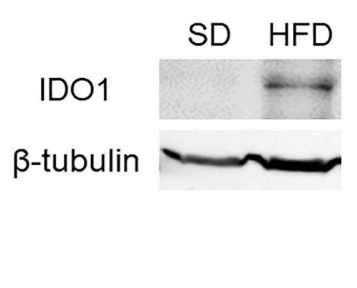

C

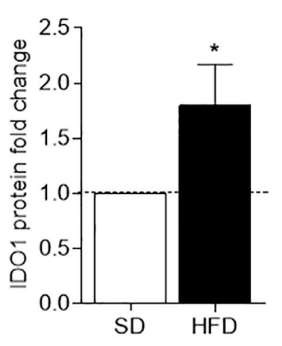

D

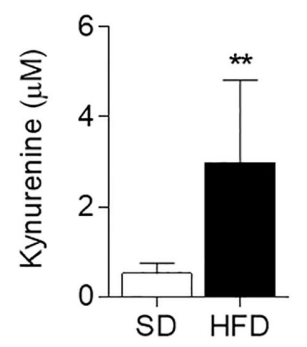

F

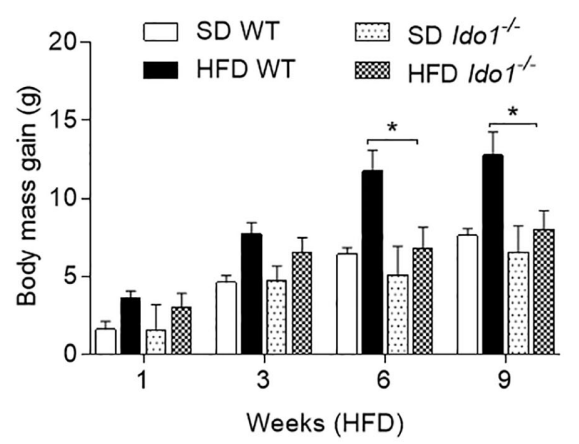

G

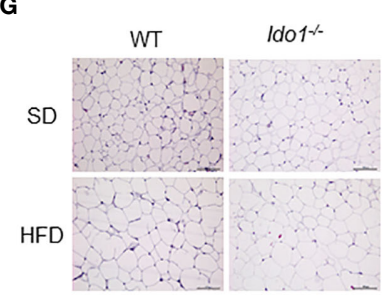

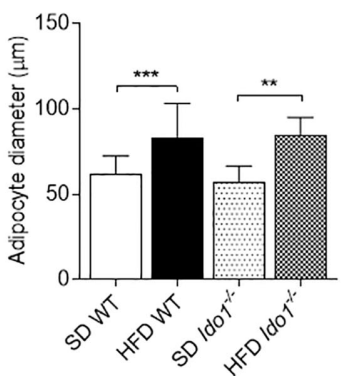

E

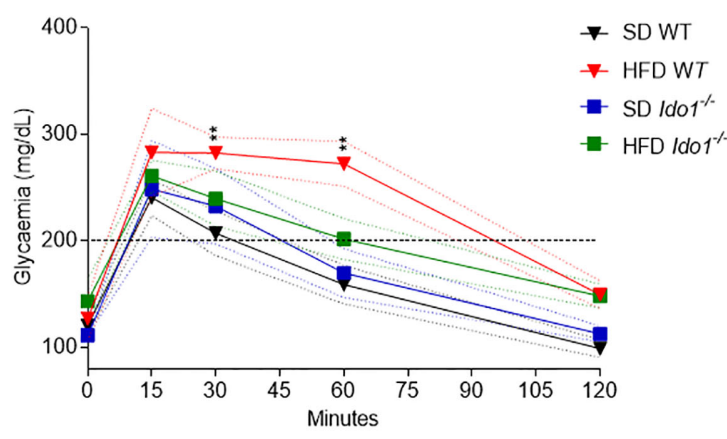

FIGURE 2 | IDO1 expression and activity in diet-induced obesity. Expression of IDO1 gene (A) and protein (B) in SVF WAT cells of HFD versus SD mice after 10 weeks of diet. (C) Quantitative analysis of immunoblots from two independent ex vivo experiments, one of which represented in (B). Data (mean \pm S.D., $n=3$ biological replicates) represent the ratio of tubulin-normalized IDO1 protein in SVF WAT from mice on HFD to that expressed in SD control counterparts. (D) Levels of Kyn (mean \pm S.D., $n=3$ biological replicates) secreted by SVF WAT cells in 24 -h culture supernatants. ${ }^{*} p<0.05$, ${ }^{* *} p<0.01$, HFD versus SD (two-tail unpaired Student's $t$ test for C, D). (E) Body weight gain of WT and $/ d 01^{-1-}$ mice throughout 9 wk of high-fat diet (HFD, $\left.n=10\right)$ treatment compared with gender- and agematched controls fed with a standard diet (SD, $n=10)$. ${ }^{*} p<0.05$, HFD WT versus HFD ldo ${ }^{-1-}$ mice, ANOVA followed by post hoc Bonferroni's method. (F) Intraperitoneal glucose tolerance test (IPGTT) after 9 wk of HFD ( $n=5$, from two independent experiments). Glycaemia (mg/dl) at different time points (0, $15,30,60$, and $120 \mathrm{~min}$ ) from the administration of glucose. (G) Hematoxylin and eosin staining of visceral WAT (left panel, scale bars are $100 \mu \mathrm{m}$.). Analysis of adipocyte diameter (right panel). ${ }^{\star \star} p<0.01,{ }^{\star \star \star} p<0.001$ HFD versus SD mice per genotype (ANOVA followed by post hoc Bonferroni's method for $\mathbf{F}$, G).

(Figure 4B) could be observed in TCZ-treated obese mice as compared to untreated obese mice. Likewise, glucose tolerance of TCZ-treated mice on HFD was indistinguishable from that of mice on standard diet (Figure 4C). TCZ effects could also be observed when the drug administration was delayed at 2 wk of feeding with HFD, when obese mice had already gained weight (Figures 4D-F). To confirm the glucose tolerance induced by TCZ treatment as a surrogate marker of insulin responsiveness, we also evaluated the insulin-induced AKT phosphorylation (pAKT) (23) in primary hepatocytes from the experimental groups shown in Figure 4D. In contrast to control mice, very low levels of pAKT could be induced in the cells from HFD-fed mice. However, the TCZ treatment completely restored insulin sensitivity by significantly increasing the ratio pAKT/AKT (Figures 4G, H). In order to see whether TCZ could also have an impact on browning, i.e., the process by which some adipocytes within WAT acquire properties of brown adipocytes ("beiging" effect), the transcript expression of uncoupling protein-1 [UCP-1; i.e., provoking energy dissipation by uncoupling respiration from ATP synthesis (24)] was evaluated. Results showed that the Ucp1 gene expression was significantly upregulated by TCZ treatment in WAT of HFD-mice as compared to untreated animals (Figure 4I). Differently from HFD-fed mice, both insulin- 
A

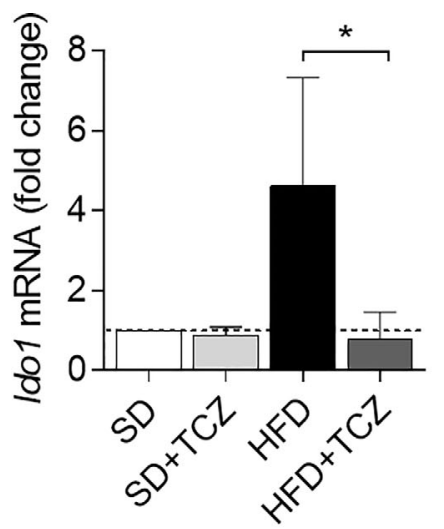

C

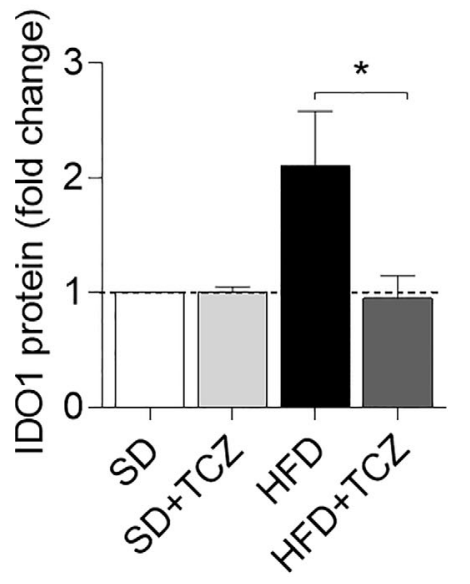

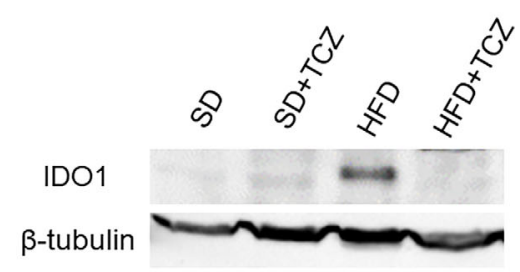

D

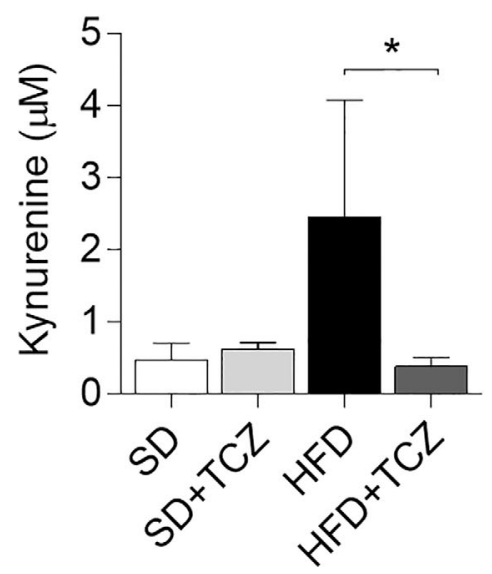

FIGURE 3 | TCZ inhibits IDO1 expression in SVF WAT. (A) Gene transcription of Ido1 in SVF WAT cells after 9 wk of diet. Data (mean \pm S.D., n = 3 biological replicates, from two independent experiments) represent the fold change expression of Gapdh-normalized transcripts in which the calibrator is represented by SVF WAT from SD-fed mice (fold change = 1; dotted line). (B) IDO1 protein expression in SVF WAT cells and quantitative analysis (C) of immunoblots from two independent ex vivo experiments, one of which shown in (B). Data (mean \pm S.D., $n=3$ biological replicates, from two independent experiments) represent the ratio of tubulin-normalized IDO1 protein expression in SVF cells from HFD-fed mice to that expressed in SVF from animals on SD ( $\mathrm{n}=3$ mice per group). (D) Levels of Kyn (mean \pm S.D., $n=3$ biological replicates, from two independent experiments) secreted by SVF WAT cells in $24-\mathrm{h}$ culture supernatants. * $\mathrm{p}<0.05$ (ANOVA followed by post hoc Bonferroni's method for $\mathbf{A}, \mathbf{C}, \mathbf{D})$.

induced phosphorylation of AKT in primary hepatocytes and induction of the Ucp1 gene resulted to be insensitive to TCZ treatment in mice fed with SD (Supplementary Figure 1).

\section{DISCUSSION}

Low-grade, chronic inflammation has also been termed metaflammation, i.e., an inflammatory state orchestrated by metabolic cells in response to excess nutrients and energy (25). In metabolic organs including the liver, pancreas, and adipose tissue, the interaction of metabolic cells with the stromal components represents an important determinant in the maintenance of tissue homeostasis, thus preventing metaflammation.

Apart from its function as an energy storage, WAT is a large metabolically and immunologically active endocrine organ composed of mature adipocytes in addition to adipose-derived stem cells, fibroblasts, endothelial cells, and a wide range of immune cells (i.e., mainly macrophages) that overall constitute the SVF WAT (26). Depending on the microenvironmental conditions, adipose-derived stem cells can differentiate into either white or brown-like adipocyte phenotypes (27). When caloric intake exceeds caloric expenditure, WAT becomes hypertrophied and heavily infiltrated by immune cells with a pro-inflammatory phenotype, causing metaflammation and obesity often associated with insulin resistance.

In animal models, it is well documented that HFD induces metaflammation (25), with the production of pro-inflammatory cytokines such as TNF- $\alpha$, IL-1 $\beta$, and IL- 6 by the adipose tissue (28). By using HFD-fed mice as an experimental model of obesity, we indeed found increased levels of those cytokines as well as of IFN- $\gamma$ in the culture supernatants of SVF WAT cells 
A

C

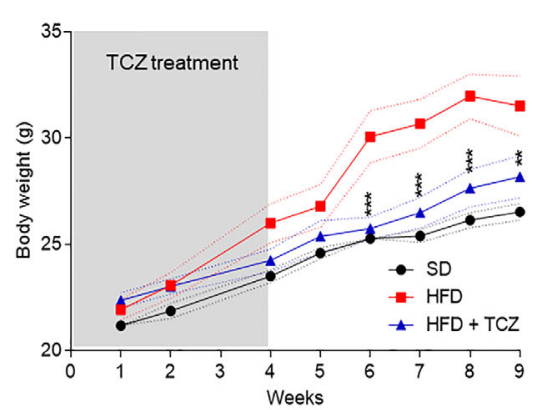

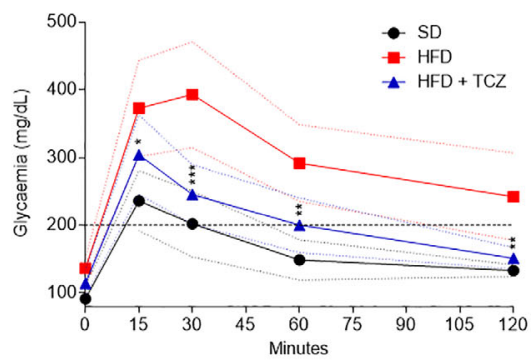

E

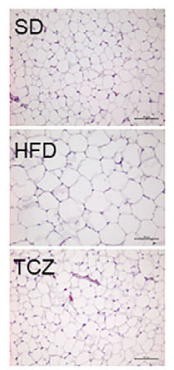

G

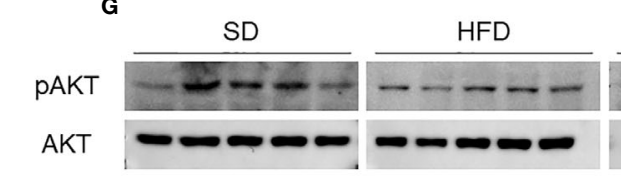

Time $(\min )$

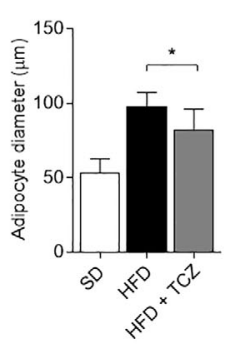

HFD

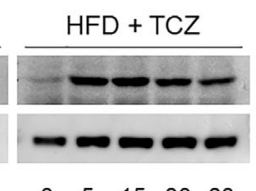

$\begin{array}{lllll}0 & 5 & 15 & 30 & 60\end{array}$

I

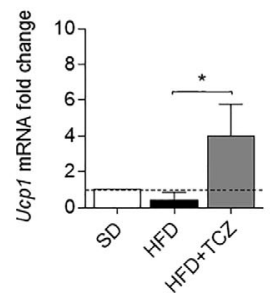

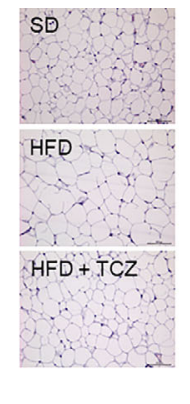
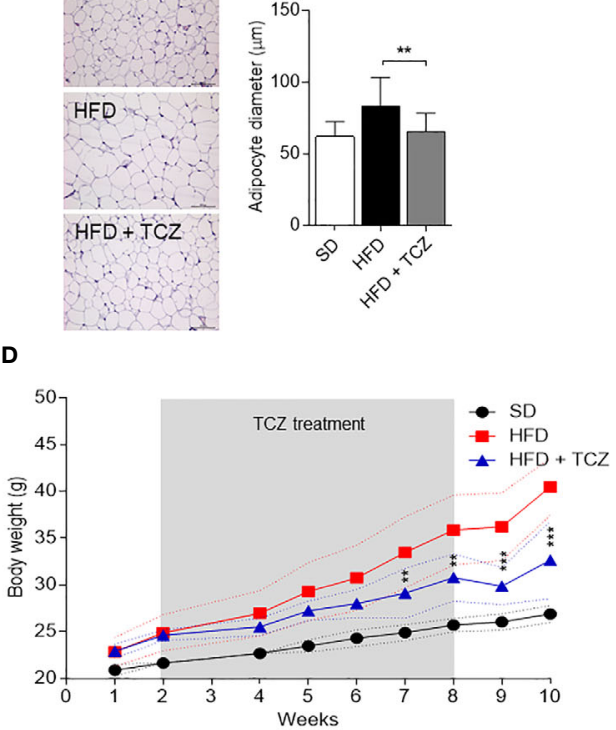

F

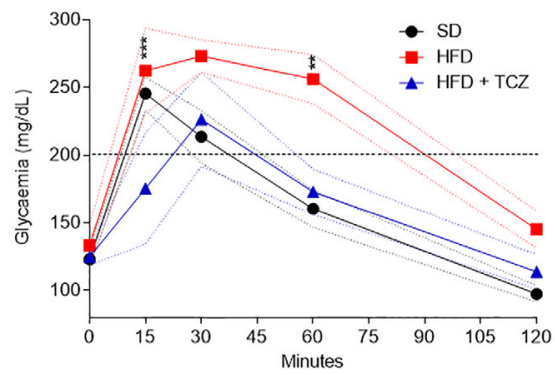

H

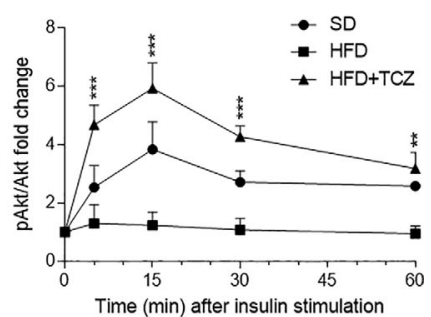

FIGURE 4 | TCZ effects in diet-induced obesity. (A, D) Body weight (g) of HFD-fed mice receiving TCZ $5 \mathrm{mg} / \mathrm{Kg}$ (HFD TCZ, $n=8)$ or saline (HFD, $n=8$ ) administered i.p. compared with gender- and age-matched controls fed with a standard diet (SD, $n=8)$. TCZ treatment started with HFD (A) or 2 wk later (D) and ended after 4 and 6 weeks, respectively, in A and D (grey box). (B, E) Hematoxylin and eosin staining of visceral WAT (left panel, scale bars of $100 \mu \mathrm{m}$.). Analysis of adipocyte diameter (right panel). (C, F) Intraperitoneal glucose tolerance test (IPGTT) at the end of TCZ treatment. Glycaemia (mg/dl) was measured at different time points $(0,15,30,60$, and $120 \mathrm{~min})$ from the administration of glucose. (G, H) Immunoblot and quantitative analysis of insulin-driven AKT phosphorylation in ex vivo hepatocytes from mice represented in (D). Data from two independent experiments (means \pm S.D., $n=3$ biological replicates per group) represent the fold change of the PAKT/AKT ratio in hepatocytes stimulated with insulin at the indicated times in which the calibrator is represented by pAKT/AKT ratio at time 0. (I) Gene transcription of Ucp1 in SVF WAT cells from mice represented in (D). Data (mean \pm S.D., $n=3$ biological replicates per group) represent the fold change expression of Gapdh-normalized transcripts in which the calibrator is represented by samples from SVF WAT from SD-fed mice (fold change $=1$; dotted line). ${ }^{*} p<0.05$, ${ }^{* \star} p<0.01,{ }^{\star \star \star} p<0.001$; HFD TCZ versus HFD (ANOVA followed by post hoc Bonferroni's method). 
from obese animals as compared to their counterparts on SD. In the same cells, such pro-inflammatory profile was accompanied by high expression and activity of IDO1, an immunometabolic enzyme involved in Trp metabolism and endowed with potent anti-inflammatory and immunoregulatory properties when expressed in DCs $(5,29)$. As hypothesized previously (13), high IDO1 expression in WAT of obese mice could be caused by higher local levels of IFN- $\gamma$, the potent inducer of the enzyme (30). Lack of IDO1 expression ameliorated the disease in terms of weight gain and glucose tolerance but not of adipocyte hypertrophy, suggesting that Trp metabolism exerts pathogenetic rather than protective effects in obesity. Mitigating effects of IDO1 depletion have been ascribed to a rewiring of host to microbiota Trp metabolism producing a protective indole derivative and not to the absence of Kyn (13), the IDO1 product known to promote arterial vessel relaxation and thus pro-inflammatory effects (31). Therefore, our data would sustain the importance of the microbiota Trp metabolism in obesity.

In addition to IFN- $\gamma$, IDO1 expression can also be upregulated in macrophages by combinations of TNF- $\alpha$, IL- $1 \beta$, and IL- 6 but not by the single cytokines (32). However, in human tumor cells, IL- 6 alone can significantly upregulate the enzyme expression (9). Because remarkable similarities between adipose expansion and growth of solid tumors have been observed (22), we evaluated the in vivo IL-6 dependency of IDO1 expression and activity in obesity. Administration of TCZ, an IL-6R blocker, to HFD-fed mice brought the levels of IDO1 transcript and protein expressions as well as Kyn production to those of control animals, thus suggesting a major role of IL- 6 rather than IFN- $\gamma$ in upregulating Trp metabolism in the obese adipose tissue. Perhaps most importantly, the TCZ treatment, either commenced at 0 or 2 wk of HFD, profoundly changed all the parameters examined by us for obesity so far, such that HFDmice were indistinguishable from their counterparts on SD. Of note, the monoclonal antibody significantly increased the expression of Ucp1, suggesting a beiging effect on the adiposederived stem cell component of SVF WAT of obese animals that may greatly contribute to the overall therapeutic effect of TCZ. Because the TCZ treatment but not IDO1 depletion also reduced adipocyte hypertrophy, our data suggested that the pathogenic role of IL-6 in the disease goes beyond IDO1 and other IL-6 -driven mechanisms may be at work.

The incidence of obesity and its serious complications, particularly cardiovascular and metabolic diseases, is steadily increasing worldwide. Unfortunately, no truly effective and safe therapeutic options are available yet. Targeting specific molecules of metaflammation with biologic drugs in the adipose tissue may provide novel opportunities of drug

\section{REFERENCES}

1. Jones SA, Jenkins BJ. Recent Insights Into Targeting the IL-6 Cytokine Family in Inflammatory Diseases and Cancer. Nat Rev Immunol (2018) 18:773-89. doi: 10.1038/s41577-018-0066-7

2. Hunter CA, Jones SA. IL-6 as a Keystone Cytokine in Health and Disease. Nat Immunol (2015) 16:448-57. doi: 10.1038/ni.3153 treatment. However, blockade of either IL-1 $\beta$ (33) or TNF- $\alpha$ (34) has shown limited success in obese patients. Besides a few number of studies in patients with rheumatoid arthritis aimed at evaluating the effects of obesity on drug effectiveness $(35,36)$, no clinical trial has been performed with TCZ in obese patients yet. In addition to provide the evidence for the existence of a pathogenetic IL-6/IDO1 axis in obesity, our data suggested that IL- 6 blockade by TCZ may represent a promising therapeutic option for obese patients.

\section{DATA AVAILABILITY STATEMENT}

The raw data supporting the conclusions of this article will be made available by the authors, without undue reservation.

\section{ETHICS STATEMENT}

The animal study was reviewed and approved by Italian Ministry of Health.

\section{AUTHOR CONTRIBUTIONS}

CO designed and supervised the study as a whole. GM and EA performed the majority of experiments. EO performed Kyn determinations. GR performed histological analyses. MB and MP helped with some experiments and provided reagents. UG and $\mathrm{CO}$ wrote the manuscript. All authors contributed to the article and approved the submitted version.

\section{FUNDING}

This work was supported by Associazione Italiana per la Ricerca sul Cancro (AIRC 2019-23084; to UG) and the Italian Ministry of Education, University, and Research (PRIN20172017BA9LM5 to CO).

\section{SUPPLEMENTARY MATERIAL}

The Supplementary Material for this article can be found online at: https://www.frontiersin.org/articles/10.3389/fimmu.2021. 713989/full\#supplementary-material

3. Johnson DE, O'Keefe RA, Grandis JR. Targeting the IL-6/JAK/STAT3 Signalling Axis in Cancer. Nat Rev Clin Oncol (2018) 15:234-48. doi: 10.1038/nrclinonc.2018.8

4. Grohmann U, Fallarino F, Puccetti P. Tolerance, DCs and Tryptophan: Much Ado About IDO. Trends Immunol (2003) 24:242-8. doi: 10.1016/S1471-4906(03)00072-3

5. Mellor AL, Munn DH. IDO Expression by Dendritic Cells: Tolerance and Tryptophan Catabolism. Nat Rev Immunol (2004) 4:762-74. doi: 10.1038/nri1457 
6. Puccetti P, Grohmann U. IDO and Regulatory T Cells: A Role for Reverse Signalling and Non-Canonical NF-KappaB Activation. Nat Rev Immunol (2007) 7:817-23. doi: 10.1038/nri2163

7. Orabona C, Pallotta MT, Grohmann U. Different Partners, Opposite Outcomes: A New Perspective of the Immunobiology of Indoleamine 2,3Dioxygenase. Mol Med (2012) 18:834-42. doi: 10.2119/molmed.2012.00029

8. van Baren N, Van den Eynde BJ. Tryptophan-Degrading Enzymes in Tumoral Immune Resistance. Front Immunol (2015) 6:34. doi: 10.3389/ fimmu.2015.00034

9. Litzenburger UM, Opitz CA, Sahm F, Rauschenbach KJ, Trump S, Winter M, et al. Constitutive IDO Expression in Human Cancer Is Sustained by an Autocrine Signaling Loop Involving IL-6, STAT3 and the AHR. Oncotarget (2014) 5:1038-51. doi: 10.18632/oncotarget.1637

10. Saltiel AR, Olefsky JM. Inflammatory Mechanisms Linking Obesity and Metabolic Disease. J Clin Invest (2017) 127:1-4. doi: 10.1172/JCI92035

11. Castoldi A, Naffah de Souza C, Camara NO, Moraes-Vieira PM. The Macrophage Switch in Obesity Development. Front Immunol (2015) 6:637. doi: 10.3389/fimmu.2015.00637

12. Calle EE, Kaaks R. Overweight, Obesity and Cancer: Epidemiological Evidence and Proposed Mechanisms. Nat Rev Cancer (2004) 4:579-91. doi: $10.1038 / \mathrm{nrc1} 408$

13. Laurans L, Venteclef N, Haddad Y, Chajadine M, Alzaid F, Metghalchi S, et al. Genetic Deficiency of Indoleamine 2,3-Dioxygenase Promotes Gut Microbiota-Mediated Metabolic Health. Nat Med (2018) 24:1113-20. doi: 10.1038/s41591-018-0060-4

14. Brandacher G, Hoeller E, Fuchs D, Weiss HG. Chronic Immune Activation Underlies Morbid Obesity: Is IDO a Key Player? Curr Drug Metab (2007) 8:289-95. doi: 10.2174/138920007780362590

15. Orabona C, Mondanelli G, Pallotta MT, Carvalho A, Albini E, Fallarino F, et al. Deficiency of Immunoregulatory Indoleamine 2,3-Dioxygenase 1 in Juvenile Diabetes. JCI Insight (2018) 3(6):e96244. doi: 10.1172/jci.insight.96244

16. Xue Y, Xu X, Zhang XQ, Farokhzad OC, Langer R. Preventing Diet-Induced Obesity in Mice by Adipose Tissue Transformation and Angiogenesis Using Targeted Nanoparticles. Proc Natl Acad Sci U S A (2016) 113:5552-7. doi: 10.1073/pnas.1603840113

17. Pallotta MT, Orabona C, Volpi C, Vacca C, Belladonna ML, Bianchi R, et al. Indoleamine 2,3-Dioxygenase is a Signaling Protein in Long-Term Tolerance by Dendritic Cells. Nat Immunol (2011) 12:870-8. doi: 10.1038/ni.2077

18. Mondanelli G, Bianchi R, Pallotta MT, Orabona C, Albini E, Iacono A, et al. A Relay Pathway Between Arginine and Tryptophan Metabolism Confers Immunosuppressive Properties on Dendritic Cells. Immunity (2017) 46:233-44. doi: 10.1016/j.immuni.2017.01.005

19. Mondanelli G, Coletti A, Greco FA, Pallotta MT, Orabona C, Iacono A, et al. Positive Allosteric Modulation of Indoleamine 2,3-Dioxygenase 1 Restrains Neuroinflammation. Proc Natl Acad Sci U S A (2020) 117:3848-57. doi: 10.1073/pnas.1918215117

20. Romani L, Fallarino F, De Luca A, Montagnoli C, D’Angelo C, Zelante T, et al. Defective Tryptophan Catabolism Underlies Inflammation in Mouse Chronic Granulomatous Disease. Nature (2008) 451:211-5. doi: 10.1038/nature06471

21. Prunet-Marcassus B, Cousin B, Caton D, Andre M, Penicaud L, Casteilla L. From Heterogeneity to Plasticity in Adipose Tissues: Site-Specific Differences. Exp Cell Res (2006) 312:727-36. doi: 10.1016/j.yexcr.2005.11.021

22. Sun K, Kusminski CM, Scherer PE. Adipose Tissue Remodeling and Obesity. J Clin Invest (2011) 121:2094-101. doi: 10.1172/JCI45887

23. Molinaro A, Becattini B, Mazzoli A, Bleve A, Radici L, Maxvall I, et al. InsulinDriven PI3K-AKT Signaling in the Hepatocyte Is Mediated by Redundant PI3Kalpha and PI3Kbeta Activities and Is Promoted by RAS. Cell Metab (2019) 29:1400-1409 e5. doi: 10.1016/j.cmet.2019.03.010

24. Nicholls DG, Locke RM. Thermogenic Mechanisms in Brown Fat. Physiol Rev (1984) 64:1-64. doi: 10.1152/physrev.1984.64.1.1
25. Hotamisligil GS. Inflammation, Metaflammation and Immunometabolic Disorders. Nature (2017) 542:177-85. doi: 10.1038/nature21363

26. Esteve Rafols M. Adipose Tissue: Cell Heterogeneity and Functional Diversity. Endocrinol Nutr (2014) 61:100-12. doi: 10.1016/j.endoen.2014.02.001

27. Liu L, Zheng LD, Donnelly SR, Emont MP, Wu J, Cheng Z. Isolation of Mouse Stromal Vascular Cells for Monolayer Culture. Methods Mol Biol (2017) 1566:9-16. doi: 10.1007/978-1-4939-6820-6_2

28. Kurylowicz A, Kozniewski K. Anti-Inflammatory Strategies Targeting Metaflammation in Type 2 Diabetes. Molecules (2020) 25(9):2224. doi: $10.3390 /$ molecules 25092224

29. Mondanelli G, Iacono A, Allegrucci M, Puccetti P, Grohmann U. Immunoregulatory Interplay Between Arginine and Tryptophan Metabolism in Health and Disease. Front Immunol (2019) 10:1565. doi: 10.3389/fimmu.2019.01565

30. Yoshida R, Imanishi J, Oku T, Kishida T, Hayaishi O. Induction of Pulmonary Indoleamine 2,3-Dioxygenase by Interferon. Proc Natl Acad Sci U S A (1981) 78:129-32. doi: 10.1073/pnas.78.1.129

31. Wang Y, Liu H, McKenzie G, Witting PK, Stasch JP, Hahn M, et al. Kynurenine Is an Endothelium-Derived Relaxing Factor Produced During Inflammation. Nat Med (2010) 16:279-85. doi: 10.1038/nm.2092

32. Fujigaki H, Saito K, Fujigaki S, Takemura M, Sudo K, Ishiguro H, et al. The Signal Transducer and Activator of Transcription 1alpha and Interferon Regulatory Factor 1 Are Not Essential for the Induction of Indoleamine 2,3-Dioxygenase by Lipopolysaccharide: Involvement of P38 MitogenActivated Protein Kinase and Nuclear Factor-KappaB Pathways, and Synergistic Effect of Several Proinflammatory Cytokines. J Biochem (2006) 139:655-62. doi: $10.1093 / \mathrm{jb} / \mathrm{mvj} 072$

33. van Asseldonk EJ, Stienstra R, Koenen TB, Joosten LA, Netea MG, Tack CJ. Treatment With Anakinra Improves Disposition Index But Not Insulin Sensitivity in Nondiabetic Subjects With the Metabolic Syndrome: A Randomized, Double-Blind, Placebo-Controlled Study. J Clin Endocrinol Metab (2011) 96:2119-26. doi: 10.1210/jc.2010-2992

34. Bernstein LE, Berry J, Kim S, Canavan B, Grinspoon SK. Effects of Etanercept in Patients With the Metabolic Syndrome. Arch Intern Med (2006) 166:902-8. doi: 10.1001/archinte.166.8.902

35. Pers YM, Godfrin-Valnet M, Lambert J, Fortunet C, Constant E, Mura T, et al. Response to Tocilizumab in Rheumatoid Arthritis Is Not Influenced by the Body Mass Index of the Patient. J Rheumatol (2015) 42:580-4. doi: 10.3899/ jrheum. 140673

36. Gardette A, Ottaviani S, Sellam J, Berenbaum F, Liote F, Meyer A, et al. Body Mass Index and Response to Tocilizumab in Rheumatoid Arthritis: A Real Life Study. Clin Rheumatol (2016) 35:857-61. doi: 10.1007/s10067-016-3183-3

Conflict of Interest: The authors declare that the research was conducted in the absence of any commercial or financial relationships that could be construed as a potential conflict of interest.

Publisher's Note: All claims expressed in this article are solely those of the authors and do not necessarily represent those of their affiliated organizations, or those of the publisher, the editors and the reviewers. Any product that may be evaluated in this article, or claim that may be made by its manufacturer, is not guaranteed or endorsed by the publisher.

Copyright (c) 2021 Mondanelli, Albini, Orecchini, Pallotta, Belladonna, Ricci, Grohmann and Orabona. This is an open-access article distributed under the terms of the Creative Commons Attribution License (CC BY). The use, distribution or reproduction in other forums is permitted, provided the original author(s) and the copyright owner(s) are credited and that the original publication in this journal is cited, in accordance with accepted academic practice. No use, distribution or reproduction is permitted which does not comply with these terms. 